\title{
Psychological distress across the deployment cycle: exploratory growth mixture model
}

\author{
Oscar A. Cabrera and Amy B. Adler
}

\section{Background}

Prior research has identified behavioural health outcomes as key sequelae to combat deployment. However, relatively little is known about differential patterns of change in depression or generalised anxiety linked to deployment to a combat zone. In this paper, we add to the existing trajectory literature and examine key predictive factors of behavioural health risk.

\section{Aims}

The primary aim is to leverage growth mixture modelling to ascertain trajectories of psychological distress, operationalised as a coherent construct combining depression and generalised anxiety, and to identify factors that differentiate adaptive and maladaptive patterns of change.

\section{Method}

Data were collected from a brigade combat team prior to a combat deployment to Afghanistan, during deployment, at immediate re-integration and approximately $2-3$ months thereafter. The main outcome was measured using the Patient Health Questionnaire Anxiety and Depression Scale (PHQ-ADS).

\section{Results}

Three latent trajectories were identified: a low-stable trajectory, a declining trajectory and a rising trajectory. Most individuals aligned with the low-stable trajectory. A conditional model using covariates measured during deployment showed that the lowstable trajectory differed consistently from the remaining trajectories on self-reported loneliness and non-combat deployment stressors

\section{Conclusions}

The examination of differential patterns of adaptation, to identify individuals at higher risk, is critical for the efficient targeting of resources. Our findings further indicate that loneliness may be a useful leverage point for clinical and organisational intervention.

\section{Keywords}

Trauma; military psychiatry; depressive disorders; anxiety disorders; comorbidity.

\section{Copyright and usage}

(c) The Author(s), 2021. Published by Cambridge University Press on behalf of the Royal College of Psychiatrists. This is an Open Access article, distributed under the terms of the Creative commons Attribution licence (http://creativecommons.org/ licenses/by/4.0/), which permits unrestricted re-use, distribution, and reproduction in any medium, provided the original work is properly cited.

\section{Background}

Prior research across a range of military samples has identified depression and generalised anxiety as key behavioural health concerns. ${ }^{1-3}$ This research has documented important covariates of depression and/or generalised anxiety, including functional impairment, ${ }^{4}$ combat exposure, ${ }^{4,5}$ non-combat deployment stressors (for example, separation from family) ${ }^{6}$ and loneliness. ${ }^{7}$

Few studies on depression and/or anxiety have attempted to ascertain the existence of differential longitudinal trajectories, where individuals cluster into discrete trajectories representing substantively different patterns of change and adaptation over time. We are not aware of any differential trajectory studies of generalised anxiety in military personnel, and we have identified only two such studies for depression. The first study ${ }^{8}$ used latent class growth analysis to examine post-deployment depression trajectories among US Army National Guard personnel who had deployed to Afghanistan. The authors reported on four trajectories: (a) a 'resistant' trajectory, showing a low-stable pattern across time; (b) a 'resilient' trajectory denoting a declining symptom pattern; (c) an 'increasing (mild)' trajectory showing rising symptoms over time, but remaining within a mild symptom range; and (d) a 'chronic-dysfunction' trajectory showing consistently higher symptomatology across time.

The second study ${ }^{9}$ used a prospective growth mixture modelling approach to examine trajectories of depression among Danish military personnel deployed to Afghanistan. The authors identified three trajectories of change: (a) a 'low-stable' trajectory with few depression symptoms; (b) a 'low-increasing' trajectory showing a significant rise in symptomatology over time, leading to severe depression at the last measurement occasion; and (c) a 'medium-fluctuating' trajectory with a pattern denoting symptomatology in the mid-range between low and severe depression. A consistent finding from these studies is that a low-stable trajectory related to depression is the most common pattern.

\section{Psychological distress and study objectives}

Given the documented strong association between depression and generalised anxiety and that these constructs may share an underlying common factor, ${ }^{10,11}$ it is important to consider both of them together as they may reflect an underlying construct of emotional distress. Examining depression and anxiety together is important not only because of their comorbidity, ${ }^{10-12}$ but because clinical therapies for these disorders tend to be transdiagnostic ${ }^{13}$ and having both depression and anxiety places individuals at greater risk of symptom chronicity and may cost more in terms of resources. ${ }^{14}$ Moreover, individuals with one set of symptoms are at risk for developing the other, suggesting that focusing only on one construct might indicate recovery when in fact the symptom picture might simply have shifted, not abated. ${ }^{15}$

Indeed, it is the covariation between depression and anxiety that inspired the development of the Patient Health Questionnaire Anxiety and Depression Scale (PHQ-ADS), ${ }^{13}$ an instrument that combines two validated scales, the Patient Health Questionnare- 9 (PHQ-9) ${ }^{16}$ and the Generalized Anxiety Disorder-7 (GAD-7), ${ }^{17}$ to assess a consolidated depression-anxiety construct. In practice, this scale has been advanced as a measure of psychological distress that may help to identify individuals at higher behavioural health risk. ${ }^{18,19}$ Consequently, the conceptual and methodological orientation of considering a combined depression/anxiety construct, as an index of psychological distress, presents an excellent opportunity to assess longitudinal variation in behavioural health risk. 


\begin{tabular}{|c|c|c|c|c|}
\hline & $\begin{array}{c}\text { Time } 1 \\
(n=760)\end{array}$ & $\begin{array}{c}\text { Time } 2 \\
(n=1142)\end{array}$ & $\begin{array}{c}\text { Time } 3 \\
(n=1142)\end{array}$ & $\begin{array}{c}\text { Time } 4, n(\%) \\
\quad(n=547)\end{array}$ \\
\hline \multicolumn{5}{|l|}{ Age, years ${ }^{c}$} \\
\hline $18-24$ & $422(55.5)$ & $556(48.7)$ & $516(45.2)$ & 239 (43.7) \\
\hline $25-29$ & $202(26.6)$ & $326(28.5)$ & $354(31.0)$ & 170 (31.1) \\
\hline 30-39 & $120(15.8)$ & $213(18.7)$ & $223(19.5)$ & $111(20.3)$ \\
\hline 40 and older & $16(2.1)$ & $44(3.9)$ & $47(4.1)$ & $26(4.8)$ \\
\hline Missing data & - & $3(0.3)$ & $2(0.2)$ & $1(0.1)$ \\
\hline \multicolumn{5}{|l|}{ Gender } \\
\hline Men & 717 (94.3) & $1070(93.7)$ & $1080(94.6)$ & $526(96.2)$ \\
\hline Women & $37(4.9)$ & $55(4.9)$ & $58(4.8)$ & $20(3.7)$ \\
\hline Missing data & $6(0.8)$ & $17(1.5)$ & $4(0.3)$ & $1(0.1)$ \\
\hline \multicolumn{5}{|l|}{ Rank $^{C}$} \\
\hline Junior enlisted & $441(58.0)$ & $611(53.5)$ & $571(50.0)$ & $254(46.4)$ \\
\hline Non-commissioned officer & 236 (31.1) & $396(34.7)$ & $442(38.7)$ & $223(40.7)$ \\
\hline Officer/warrant officer & $80(10.5)$ & $126(11.0)$ & $127(11.1)$ & $70(12.8)$ \\
\hline Missing data & $3(0.4)$ & $9(0.8)$ & $2(0.2)$ & - \\
\hline
\end{tabular}

To date, no study has examined latent trajectory heterogeneity in psychological distress, as defined here (i.e. as a combined depression-anxiety construct). The present study aims to close this gap. First, this study utilises growth mixture modelling to measure heterogeneity in the course of psychological distress, which will facilitate the identification of differential risk groups. Second, this study ascertains the modal latent trajectory pattern (i.e. the pattern with which the majority of individuals align). In this way, we will be able to better understand the most common experience of service members. Third, this study includes predictors of assignment-to-trajectory included in the depression trajectory studies cited above (i.e. combat exposure and rank), as well as important predictors not previously included (i.e. loneliness and non-combat deployment stressors). Finally, these predictors are all measured during deployment, enabling an assessment of important covariates temporally linked to the combat experience.

\section{Method}

\section{Participants}

Survey data were collected from soldiers serving in a brigade-sized combat unit deployed to Afghanistan in the 2013-2014 time frame, as part of a larger study of health and resilience across the deployment cycle. ${ }^{20,21}$ Data on the PHQ-9 and the GAD-7 were only available for four time points. Thus, the final analytic sample $(n=1142)$ consisted of those soldiers who deployed with the unit to Afghanistan, who provided complete data on the outcome measure at time 2 (during deployment), and who provided complete data on at least one of the other three sessions: (a) time 1 (pre-deployment); (b) time 3 (initial post-deployment phase, approximately 1 month after returning home); and (c) time 4 (approximately 2-3 months post-deployment). This selection was affected to maximise sample sizes at time 2, where the time code for model intercept was set because of the intended use of predictors co-occurring with the unit's deployment to Afghanistan. Demographic characteristics of the analytic sample from each time period are presented in Table 1. Demographic characteristics of the sample not selected for analysis are presented in Supplementary Table 1 available at https://doi.org/ 10.1192/bjo.2021.50.

Soldiers were tracked within their units, and some participant loss was expected. Specifically, attrition was predictable given that military service is characterized by substantial geographic mobility, and that some soldiers would be unable to participate in specific data collection occasions because of conflicting duty assignments. Indeed, as shown in Table 1, there was participant loss over time, although it is important to note that the loss documented in this study is consistent with similar attrition reported in other studies that have examined units across their deployment cycle. ${ }^{22,23}$

The study was conducted under a human-use protocol approved by the Institutional Review Board of the Walter Reed Army Institute of Research (WRAIR). The investigators have adhered to the policies for protection of human participants as prescribed in AR 70-25. Participants provided informed consent prior to enrolment, with $92 \%$ consenting to participate.

\section{Measures}

The outcome was measured using the 16-item PHQ-ADS. ${ }^{13}$ As noted previously, the PHQ-ADS combines depression and anxiety items from the PHQ- ${ }^{16}$ and the GAD-7, ${ }^{17}$ and the psychometric properties of the PHQ-ADS as a combined measure have been explored and validated with various samples. Items were rated in terms of the past month on a four-point scale ('not at all' to 'nearly every day'). The possible range was from 0 to 48 . For reference, cut-off scores for mild, moderate, and severe categories are 10, 20 and 30 on this scale. Coefficient alpha was 0.93, 0.93, 0.92 , and 0.92 across the four time points.

Combat exposure (time 2) was measured using 22 items adapted from the WRAIR Combat Exposure Scale. ${ }^{2,4}$ Sample items included: 'receiving small arms fire'; 'handling or uncovering human remains'. On each question, the participant was asked the number of times they had experienced that event on combat deployments since 9/11 ('never' to 'five or more times'). To simplify analyses, response options were dichotomised ('yes'/'no') and the resulting items were summed to create the combat exposure construct. Combat exposure items were considered formative, not reflective, so coefficient alpha for this measure was not calculated. ${ }^{24}$

Loneliness (time 2) was measured with a nine-item scale adapted from the UCLA Loneliness Scale. ${ }^{25,26}$ A list of items related to loneliness were presented, and respondents were asked how often they felt that that aspect of loneliness applied to them. Sample items included: 'how often to do you feel that you lack companionship?'; 'how often to do you feel close to people?'; etc. The response scale ranged from 1 ('never') to 4 ('always'). Positively worded items were reverse-coded, 


\begin{tabular}{|lcrrr|}
\hline & One class & Two classes & Three classes & Four classes (not selected) \\
\hline Loglikelihood & -11647.36 & -11429.54 & -11332.02 & -11277.04 \\
Akaike information criterion & 23312.72 & 22883.09 & 22694.03 & 22590.07 \\
Bayesian information criterion & 23358.08 & 22943.58 & 22769.64 & 22680.80 \\
Sample size adjusted-Bayesian information criterion & 23329.50 & 22905.46 & 22722.00 & 22623.63 \\
Entropy & $\mathrm{N} / \mathrm{A}$ & 0.92 & 0.90 & 0.89 \\
Lo-Mendell-Rubin likelihood ratio test, $P$ & $\mathrm{~N} / \mathrm{A}$ & $<0.001$ & $<0.01$ & 0.37 \\
Vuong-Lo-Mendell-Rubin likelihood ratio test, $P$ & $\mathrm{~N} / \mathrm{A}$ & $<0.001$ & $<0.01$ & 0.39 \\
Bootstrap likelihood ratio test, $P$ & $\mathrm{~N} / \mathrm{A}$ & $<0.001$ & $<0.001$ & $<.001$ \\
Smallest class, \% & $\mathrm{N} / \mathrm{A}$ & 12.0 & 7.1 & 4.3 \\
NA, not applicable. & & & & \\
\hline
\end{tabular}

such that higher scores for all items indicated stronger feelings of loneliness. Cronbach's alpha for this scale was 0.90 .

Non-combat deployment stressors were measured using 12 items from a scale developed by the WRAIR, and used in behavioural health assessments and published studies with deployed troops. ${ }^{5,27}$ Respondents were asked to rate how much 'trouble or concern' had been caused by a list of stressors. Examples of stressors that individuals could endorse included the following: 'being separated from family'; 'lack of privacy or personal space'. Response options ranged from 1 ('very low') to 5 ('very high') and 6 ('does not apply'); this last value was set to missing and items were summed. Cronbach's alpha for the scale was 0.86 . Supplementary Table 2 shows the array of non-combat deployment stressors, with associated endorsement rates.

Rank (time 2) was measured using one item embedded within the demographics section of the survey. Categories for this variable were 'junior enlisted', 'non-commissioned officer (NCO)', and 'officer/warrant officer'. To facilitate group contrasting, this variable was dichotomised 'junior enlisted' and 'other'.

\section{Analytic plan}

Growth mixture analyses were executed following recommendations to conduct initial enumeration of trajectories within an unconditional model (i.e. omitting covariates), with subsequent integration of covariates in a final, conditional model. ${ }^{28-33}$ As noted earlier, the model intercept was set at time 2, during deployment. Missing data were expected and were the result of random variation in the military re-assignment cycle and/or unit operational requirements during data collections. Thus, data were assumed to be missing-atrandom (MAR) and full information maximum likelihood was employed in all analyses. This method has been shown to yield unbiased parameter estimates under the MAR assumption. ${ }^{34,35}$

For unconditional model selection, three major criteria were used: (a) interpretability and parsimony; (b) Bayesian information criterion (BIC) and the bootstrap likelihood ratio test (BLRT); and (c) entropy. With regard to statistical criteria, prior simulation work ${ }^{36}$ has found that BIC performed better than other information criteria in identifying the correct number of classes, and the BLRT held the advantage in correct class enumeration when compared to other likelihood ratio test indices. Previously published guidelines were used to ascertain the magnitude of BIC changes. ${ }^{37}$ All analyses were carried out in MPlus, Version 8.2. ${ }^{38}$

\section{Results}

\section{Unconditional model}

Following definition of model selection criteria, we proceeded to estimation of the unconditional model. Here, attempts to fit a quadratic term encountered convergence errors, suggesting simpler patterns fit the data better. Therefore, we modified the model to estimate intercept and first-order slope terms only. This model configuration converged normally, and it showed that the two-class model represented an improvement over the single-class model, while the three-class model provided a better solution than the two-class model. With extraction of four trajectories, we encountered an issue involving the first selection criterion, interpretability and parsimony. Specifically, the fourth trajectory was very similar in character to one of the trajectories extracted in the three-class model: it appeared that the algorithm segmented one trajectory into a slightly lower and slightly higher variant, with the same slope. This specific type of trajectory segmentation has been identified in prior research as a warning of potential over-extraction, with the recommendation to explore solutions with fewer trajectories as a more defensible approach to model selection. ${ }^{33}$ Therefore, mindful of the violation of parsimony and the potential for over-extraction, we selected the three-class model as the final unconditional model. Table 2 displays fit statistics defining one- through four-trajectory models. Indices for analyses of depression and anxiety, as separate constructs, are presented in Supplementary Tables 3 and 4, respectively.

Entropy for the unconditional model was well within the acceptable range: $0.90 .^{33}$ The largest trajectory (83.8\% of respondents, 'low-stable') consisted of a pattern of psychological distress marked by low scores at time $2(b=3.46$, s.e. $=0.19, P<0.001)$, with negligible change in absolute scores over time $(b=-0.33$, s.e. $=0.14, P<0.05)$. The next largest trajectory $(9.1 \%$ of respondents, 'increasing') aligned with a pattern of moderate psychological distress at time $2(b=14.16$, s.e. $=0.72, P<0.001)$, occurring jointly with large increases in distress scores over time $(b=7.75$, s.e. $=0.77$, $P<0.001$ ). The third trajectory (roughly $7.1 \%$ of respondents, 'decreasing') showed higher psychological distress at time 2 ( $b=$ 16.55 , s.e. $=1.11, P<0.001)$, followed by large decreases in distress scores over time $(b=-7.69$, s.e. $=1.65, P<0.001)$. The averaged probabilities for assignment-to-trajectory were: $97 \%$ for the 'lowstable' trajectory; $84 \%$ for the 'decreasing' trajectory; and $90 \%$ for the 'increasing' trajectory.

Figure 1 shows the three trajectories, as extracted from the unconditional model.

\section{Conditional model}

For conditional model analyses, four time-invariant covariates were added in sequence. This model encountered convergence issues with the addition of a third covariate, which was resolved by constraining the slope variance (this parameter estimate was small and non-significant). As expected from this minor adjustment, the trajectories remained consistent with those defined in the previous step (see Fig. 1). There was a minor increase in entropy (0.91). The mixture changed slightly: $83.7 \%$ for low-stable; $9.8 \%$ for the 'increasing' trajectory; and $6.5 \%$ for the 'decreasing' trajectory. 


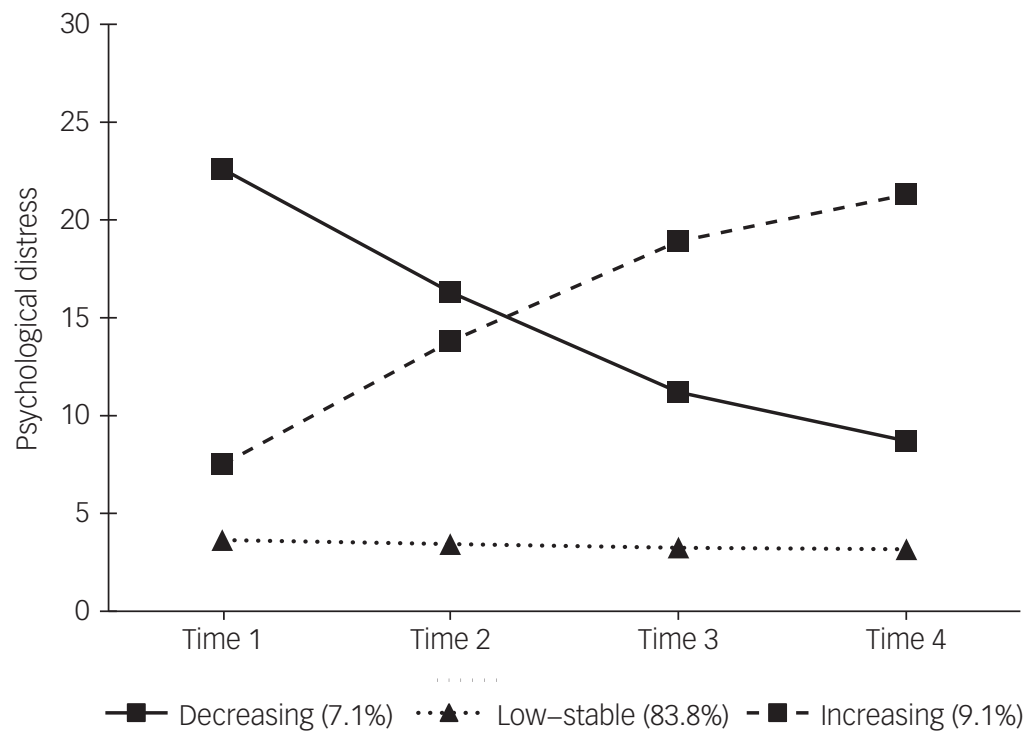

Fig. 1 Trajectories extracted from unconditional model

\begin{tabular}{|c|c|c|c|c|}
\hline & Combat exposure, OR $(95 \% \mathrm{Cl})$ & Non-combat deployment stressors, OR (95\% Cl) & Loneliness, OR (95\% Cl) & Rank, OR $(95 \% \mathrm{Cl})$ \\
\hline Low-stable & Reference & Reference & Reference & Reference \\
\hline Increasing & $1.03(0.98-1.08)$ & $1.04(1.01-1.07)$ & $1.09(1.05-1.14)$ & $0.831(0.42-1.66)$ \\
\hline Decreasing & $1.04(0.99-1.10)$ & $1.12(1.05-1.19)$ & $1.16(1.07-1.27)$ & $0.36(0.13-0.96)$ \\
\hline
\end{tabular}

Subsequently, we proceeded to examine covariate effects. These tests yielded interesting results. First, combat exposure did not differentiate trajectories. There were significant contrasts for the remaining three predictors, focused on differences between the 'low-stable' trajectory and the other two trajectories. Specifically, for every 1-point increase in reported non-combat deployment stressors, the odds of assignment to the 'increasing' and 'decreasing' trajectories rose significantly versus assignment to the 'low-stable' 'trajectory. With higher reported loneliness, the odds of assignment to the 'increasing' and 'decreasing' trajectories also increased significantly versus assignment to the 'low-stable' trajectory. In addition, with increase in rank, the odds of assignment to the 'decreasing' category versus assignment to the 'low-stable' category fell significantly, although the effect was weak. Finally, there were no significant differences between the 'increasing' and 'decreasing' trajectories on any of the covariates chosen for this study.

Table 3 shows the odds ratio (and 95\% CIs) associated with assignment-to-trajectory when compared with the odds of assignment to the 'low-stable' trajectory, as a function of a 1-unit increase in the value of each predictor.

\section{Discussion}

\section{General findings}

The literature on differential response trajectories associated with military deployment is notable for its focus on post-traumatic stress. Less attention has been paid to depression and generalised anxiety, although two studies ${ }^{8,9}$ examined depression from the vantage point of latent trajectory modelling. The present study was designed to expand this area of study by examining trajectories of psychological distress, constituted as consolidated responses for depression and generalised anxiety and using the PHQ-ADS to operationalise the construct. This approach was selected in order to model a broad-based index of behavioural health risk, thereby maximising the utility of our findings for clinicians and end-users tasked with identifying military personnel at higher risk of negative sequelae across the deployment cycle. Analyses yielded three trajectories of change: a 'low-stable' pattern denoting low scores across time; a 'decreasing' trajectory showing significant reductions in psychological distress scores; and an 'increasing' trajectory indicative of a significant rise in psychological distress. Unlike other trajectory studies, there was no 'chronic' trajectory of psychological distress. This lack of a chronic subtype might be the result of greater fluctuations in depression and anxiety symptomatology over the course of time and/or may be indicative of sample-specific characteristics (i.e. reflecting health and resilience in the analytic sample).

A few conclusions can also be gleaned from these results. First, these trajectories re-capitulate some of the findings reported in the two depression trajectory studies cited above. Specifically, our study replicates two of the three trajectories from a study of Danish personnel deployed to Afghanistan, ${ }^{9}$ and three of the four patterns reported in a post-deployment study of National Guard personnel. ${ }^{8}$ We recognise that differences between our study and these other studies may be because we used a combined depression-anxiety construct rather than focusing on depression alone.

Second, the most common trajectory in this study was a lowstable pattern denoting relatively low levels of psychological distress throughout deployment, which is consistent with the depression trajectory studies cited above and with prior theoretical work. ${ }^{39,40}$ This finding bolsters the conclusion that low expression of this outcome is the modal response pattern across the deployment cycle. 
Third, combat-related events did not differentiate among the patterns of adaptation we identified. This replicates results reported in one of the depression studies cited above, ${ }^{9}$ where the combat exposure construct was not a significant predictor of assignmentto-trajectory for depression. Critically, however, non-combat deployment stressors differentiated the low-stable trajectory from the remaining two trajectories. The consistent ability of noncombat deployment stressors to differentiate trajectories, above and beyond the influence of combat exposure, extends findings pioneered elsewhere, ${ }^{5,6,27}$ and points to the need to model a wider array of modifiable stressors in the deployed environment that may have an impact on behavioural health outcomes.

Fourth, loneliness, measured during deployment, emerged as a significant predictor of trajectory. This finding reinforces evidence of the deleterious effects of loneliness on health found in civilian ${ }^{41}$ and military studies. ${ }^{7,42}$ This finding also suggests the need for future research to identify what variables can be leveraged to reduce the risk of loneliness, including team-based interventions, ${ }^{43}$ the use of peer supports (for example Trauma Risk Management, or TRiM $)^{44}$ and leadership targeting specific health-related behaviours. ${ }^{20}$

Taken together, the results suggest that although the majority of individuals returning from combat do not report psychological distress, there are subgroups of soldiers at risk for increasing symptomatology. Identifying this subgroup is critical for developing early interventions that can be used to flatten the rising trajectory. In contrast, individuals prior to deployment who are reporting high levels of psychological distress can be targeted for interventions to facilitate their decrease in symptoms. In both cases, such interventions can include formal treatment and/or training designed to support healthy adaptation through peer-based support and targeted leadership. ${ }^{20}$ Importantly, considering depression and anxiety through a lens of comorbidity is essential for addressing the fact that one set of symptoms may lead to another, ${ }^{15}$ that having both sets of symptoms leads to greater chronicity ${ }^{12}$ and this combination of symptoms is associated with greater resource utilisation. ${ }^{14}$ Thus, it is of benefit to consider both sets of symptoms together, rather than in isolation.

\section{Limitations}

Three major study limitations need to be acknowledged. First, although the sample size for this analysis was fairly robust, larger sample sizes may be useful, especially given that the objective of growth mixture modelling is to dis-aggregate the overall outcome distribution into smaller components. Second, this analysis was limited to one combat deployment and may not generalise to other, more kinetic deployments or deployments that involve humanitarian response (for example, responding to a pandemic). Finally, data to create the PHQ-ADS measure were only available for four time points, and covered only up to 3 months after return from deployment. This provides a limited vantage point from which to examine the course of this phenomenon. With a longer time frame and additional time points, examination of more complex patterns of change becomes feasible, and such data may provide a better assessment of the evolution of psychological distress. Inclusion of time-varying covariates also becomes possible with additional time points.

\section{Implications}

We hope that these findings will encourage further examination of trajectories of outcomes related to the deployment cycle in order to better understand the impact of a combat deployment on the adjustment of service members. Latent trajectory modelling with other related outcomes (for example, functional impairment, sleep problems, optimism) can be useful in understanding the way in which groups of individuals respond to this type of high-stakes occupational demand. Potential extensions of this study include examinations that extend analysis of distress across a longer time frame, preferably following units over years, to assess the lasting impact of deployment on the course of this phenomenon.

With regard to intervention development, latent trajectory studies such as this provide a better vantage point from which to identify potential targets of intervention. As an example, findings in this study about the influence of non-combat deployment stressors may represent the core of new clinical and/or organisational interventions. That is, our results suggest potential avenues for clinicians, first-line supervisors and senior military leaders to consider in establishing environmental conditions that may support the mental health, and ultimately the functioning, of deployed military personnel. For example, stressors that reflect living conditions (such as 'lack of privacy or personal space') could be addressed in a mature theatre of operations, and stressors that reflect uncertainty (such as 'continuous operations') could be addressed through establishing parameters to manage expectations. Likewise, our robust findings regarding the covariation between loneliness and psychological distress may provide a fruitful avenue for the development of clinical interventions that target this specific risk factor.

Thus, with each application of latent trajectory modelling, opportunities arise to improve our ability to have a positive and proactive impact on the health and performance of military personnel. The fact that these risk factors were assessed during deployment also suggest that interventions might be considered either prior to or during the actual deployment, which in turn may influence subsequent post-deployment adjustment. Existing behavioural health resources such as combat operational stress control teams ${ }^{45}$ may be able to integrate key prevention strategies and implement training with leaders or with units during critical phases. Finally, it is important to ascertain if these trajectories are directly related to the deployment cycle or if they also reflect adjustment in garrison life, as well.

Oscar A. Cabrera (D), U.S. Army Medical Research Directorate-West, Walter Reed Army Institute of Research, Joint Base Lewis-McChord, USA; Amy B. Adler (DD, Walter Reed Army Institute of Research, USA

Correspondence: Oscar A. Cabrera. Email: oscar.a.cabrera2.mil@mail.mil

First received 8 Sep 2020, final revision 15 Jan 2021, accepted 23 Mar 2021

\section{Supplementary material}

Supplementary material is available online at https://doi.org/10.1192/bjo.2021.50.

\section{Data availability}

The data that support the findings of this study are available on request from the corresponding author. The data are not publicly available because of restrictions related to human participants protection requirements within the institute.

\section{Acknowledgements}

We thank Jeffrey Thomas, Rachel Eckford and Carla Kreilein for their work on this study. Material has been reviewed by the Walter Reed Army Institute of Research. There is no objection to its presentation and/or publication. The opinions or assertions contained herein are the private views of the author, and are not to be construed as official, or as reflecting true views of the Department of the Army or the Department of Defense.

\section{Author contributions}

Authors meet all criteria for authorship. The primary author developed the initial manuscript concept, drafted the manuscript, analysed the data, interpreted results, approved the final version, and is accountable for accuracy and integrity. The second author contributed to study design and data collection, refinement of the manuscript concept, revisions of manuscript drafts for critical intellectual content, approval of final version, and is accountable for accuracy and integrity. 


\section{Funding}

Data used in this manuscript were drawn from a larger study that was funded by the Military Operational Medicine Research Program, US Army Medical Research and Development Command.

\section{Declaration of interest}

None.

\section{References}

1 Black DW, Carney CP, Peloso PM, Woolson RF, Schwartz DA, Voelker MD, et al. Gulf war veterans with anxiety: prevalence, comorbidity, and risk factors. Epidemiology 2004; 15: 135-42.

2 Hoge CW, Castro CA, Messer SC, McGurk D, Cotting DI, Koffman RL. Combat duty in Iraq and Afghanistan, mental health problems, and barriers to care. N Engl J Med 2004; 351: 13-22.

3 Thomas JL, Wilk JE, Riviere LA, McGurk D, Castro CA, Hoge CW. Prevalence of mental health problems and functional impairment among active component and National Guard soldiers 3 and 12 months following combat in Iraq. Arch Gen Psychiatry 2010; 67: 614-23

4 Wright KM, Cabrera OA, Adler AB, Bliese PD. Functional impairment as a variable in adjustment post-combat. Mil Psychol 2013; 25: 545-56.

5 Thomas JL, Britt TW, Odle-Dusseau H, Bliese PD. Dispositional optimism buffers combat veterans from the negative effects of warzone stress on mental health symptoms and work impairment. J Clin Psychol 2011; 67: 866-80.

6 Russell DW, Russell CA. The evolution of mental health outcomes across a combat deployment cycle: a longitudinal study of a Guam-based national guard unit. PLOS One 2019; 14: e0223855.

7 Williams RA, Hagerty BM, Yousha SM, Hoyle KS, Oe H. Factors associated with depression in navy recruits. J Clin Psychol 2002; 58: 323-37.

8 Sampson L, Cohen GH, Calabrese JR, Fink DS, Tamburrino M, Liberzon I, et al. Mental health over time in a military sample: the impact of alcohol use disorder on trajectories of psychopathology after deployment. JTrauma Stress 2015; 28: 547-55.

9 Karstoft K-I, Nielsen AB, Armour C, Vedtofte MS, Andersen SB. Trajectories of depression symptoms from pre-to post-deployment: does previous trauma predict symptom increase? J Affect Disord 2020; 266: 120-7.

10 Breier A, Charney DS, Heninger GR. The diagnostic validity of anxiety disorders and their relationship to depressive illness. Am J Psychiatry 1985; 142: 787-97.

11 Moffitt TE, Harrington H, Caspi A, Kim-Cohen J, Goldberg D, Gregory AM, et al. Depression and generalized anxiety disorder: cumulative and sequential comorbidity in a birth cohort followed prospectively to age 32 years. Arch Gen Psychiatry 2007; 64: 651-60.

12 Löwe B, Spitzer RL, Williams JB, Mussell M, Schellberg D, Kroenke K. Depression, anxiety and somatization in primary care: syndrome overlap and functional impairment. Gen Hosp Psychiatry 2008; 30: 191-9.

13 Kroenke K, Wu J, Yu Z, Bair M, Kean J, Stump T, et al. Patient health questionnaire anxiety and depression scale: initial validation in three clinical trials. Psychosom Med 2016; 78: 716-27.

14 McLaughlin TP, Khandker RK, Kruzikas DT, Tummala R. Overlap of anxiety and depression in a managed care population: prevalence and association with resource utilization. J Clin Psychiatry 2006 Aug; 67: 1187-93.

15 Lamers F, van Oppen P, Comijs HC, Smit JH, Spinhoven P, van Balkom AJ, et al. Comorbidity patterns of anxiety and depressive disorders in a large cohort study: the Netherlands Study of Depression and Anxiety (NESDA). J Clin Psychiatry 2011; 72: 341-8.

16 Spitzer RL, Kroenke K, Williams JB. Validation and utility of a self-report version of prime-md: the PHQ primary care study. Primary Care Evaluation of Mental Disorders. Patient Health Questionnaire. JAMA 1999; 282: 1737-44.

17 Spitzer RL, Kroenke K, Williams JB, Lowe B. A brief measure for assessing generalized anxiety disorder: the gad-7. Arch Intern Med 2006; 166: 1092-7.

18 Chilcot J, Hudson JL, Moss-Morris R, Carroll A, Game D, simpson A, et al. Screening for psychological distress using the patient health questionnaire anxiety and depression scale (PHQ-ADS): initial validation of structural validity in dialysis patients. Gen Hosp Psychiatry 2018; 50: 5-9.

19 Timmins L, Rimes KA, Rahman Q. Minority stressors, rumination, and psychological distress in monozygotic twins discordant for sexual minority status. Psychol Med 2018; 48: 1705-12.

20 Adler AB, Saboe KN, Anderson JA, Sipos ML, Thomas JL. Behavioral health leadership: new directions in occupational mental health. Curr Psychiatry Rep 2014; 16: 484.
21 Adrian AL, Thomas JL, Adler AB. Soldiers and leaders with combat experience: unit health and climate. Psychiatry 2018; 81: 1-17.

22 Adler AB, Bliese PD, McGurk D, Hoge CW, Castro CA. Battlemind debriefing and battlemind training as early interventions with soldiers returning from Iraq: randomization by platoon. J Consult Clin Psychol 2009; 77: 928-40.

23 Adler AB, Britt TW, Castro CA, McGurk D, Bliese PD. Effect of transition home from combat on risk-taking and health-related behaviors. I Trauma Stress 2011; 24: 381-9.

24 Castro CA, Adler AB, McGurk D, Bliese PD. Mental health training with soldiers four months after returning from Iraq: randomization by platoon. J Trauma Stress 2012; 25: 376-83.

25 Russell DW. UCLA loneliness scale (version 3): reliability, validity, and factor structure. J Pers Assess 1996; 66: 20-40.

26 Hawkley LC, Hughes ME, Waite L, Masi CM, Thisted RA, Cacioppo JT. From social structural factors to perceptions of relationship quality and loneliness: the Chicago health, aging, and social relations study. J Gerontol B Psychol SCi Soc Sci 2008; 63: S375-84.

27 Office of The Surgeon General United States Army Medical Command, Office of the Command Surgeon Headquarters US Army Central Command (USCENTCOM), Office of the Command Surgeon US Forces Afghanistan (USFOR-A). US Army Mental Health Advisory Team 9 Report. US Army, 2013.

28 Bauer DJ. Observations on the use of growth mixture models in psychological research. Multivariate Behav Res 2007; 42: 757-86.

29 Berlin KS, Parra GR, Williams NA. An introduction to latent variable mixture modeling (part 2): Iongitudinal latent class growth analysis and growth mixture models. J Pediatr Psychol 2014; 39: 188-203.

30 Jung T, Wickrama KAS. An introduction to latent class growth analysis and growth mixture modeling. Soc Personal Psychol Compass 2008; 2: 302-17.

31 Muthén B. Latent variable analysis: growth mixture modeling and related techniques for Iongitudinal data. In The SAGE Handbook of Quantitative Methodology for the Social Sciences (ed. D Kaplan): 345-68. SAGE, 2004.

32 Petras $\mathrm{H}$, Masyn $\mathrm{K}$. General growth mixture analysis with antecedents and consequences of change. In Handbook of Quantitative Criminology (eds AR Piquero, D Weisburd): 69-100. Springer, 2010.

33 Ram N, Grimm KJ. Growth mixture modeling: a method for identifying differences in longitudinal change among unobserved groups. Int J Behav Dev 2009; 33: 565-76

34 Neale MC. Individual fit, heterogeneity, and missing data in multigroup structural equation modeling. In Modeling Longitudinal and Multilevel Data: Practical Issues, Applied Approaches, and Specific Examples (eds TD Little, KU Schnabel, JE Baumert): 224-42. Lawrence Erlbaum Associates, 2000.

35 Preacher KJ, Wichman AL, MacCallum RC, Briggs NE. Latent Growth Curve Modeling. Sage, 2008

36 Nylund KL, Asparouhov T, Muthén BO. Deciding on the number of classes in latent class analysis and growth mixture modeling: a Monte Carlo simulation study. Struct Equ Modeling 2007; 14: 535-69.

37 Kass R, Raftery A. Bayes factor and model uncertainty. J Am Stat Assoc 1995; 90: 773-95.

38 Muthén L, Muthén B. Mplus User's Guide (8th edn): 944. Muthen \& Muthen, 1998-2017.

39 Bonanno GA. Resilience in the face of potential trauma. Curr Dir Psychol Sci 2005; 14: 135-8.

40 Bonnano G. Loss, trauma and human resilience: conceptual and empirical connections and separateness. Am Psychol 2004; 59: 20-8.

41 Cacioppo S, Grippo AJ, London S, Goossens L, Cacioppo JT. Loneliness: clinical import and interventions. Perspect Psychol Sci 2015; 10: 238-49.

42 Cacioppo JT, Cacioppo S, Adler AB, Lester PB, McGurk D, Thomas JL, et al. The cultural context of loneliness: risk factors in active duty soldiers. J Soc Clin Psychol 2016; 35: 865-82.

43 Cacioppo JT, Adler AB, Lester PB, McGurk D, Thomas JL, Chen $\mathrm{H}-\mathrm{Y}$, et al. Building social resilience in soldiers: a double dissociative randomized controlled study. J Pers Soc Psychol 2015; 109: 90-105.

44 Whybrow D, Jones N, Greenberg N. Promoting organizational well-being: a comprehensive review of trauma risk management. Occup Med 2015; 65 $331-6$

45 Lewis SJ. Combat stress control: putting principle into practice. In Military Life: The Psychology of Serving in Peace and Combat: Operational Stress, vol. 2. Operational Stress (eds AB Adler, CA Castro, TW Britt): 121-40. Praeger Security International, 2006. 